\title{
La femme au rouleau : images de femmes lettrées dans le monde grec antique
}

\section{Madalina Dana}

\section{Q OpenEdition}

1 Journals

Édition électronique

URL : http://journals.openedition.org/rbnu/1148

DOI : $10.4000 /$ rbnu. 1148

ISSN : 2679-6104

\section{Éditeur}

Bibliothèque nationale et universitaire de Strasbourg

\section{Édition imprimée}

Date de publication : 1 novembre 2016

Pagination : 16-25

ISBN : 9782859230630

ISSN : 2109-2761

\section{Référence électronique}

Madalina Dana, « La femme au rouleau : images de femmes lettrées dans le monde grec antique », La Revue de la BNU [En ligne], 14 | 2016, mis en ligne le 01 janvier 2020, consulté le 11 décembre 2020. URL : http://journals.openedition.org/rbnu/1148; DOI : https://doi.org/10.4000/rbnu.1148

\section{(c) (i) (2)(2)}

La Revue de la BNU est mise à disposition selon les termes de la Licence Creative Commons Attribution - Pas d'Utilisation Commerciale - Partage dans les Mêmes Conditions 4.0 International. 


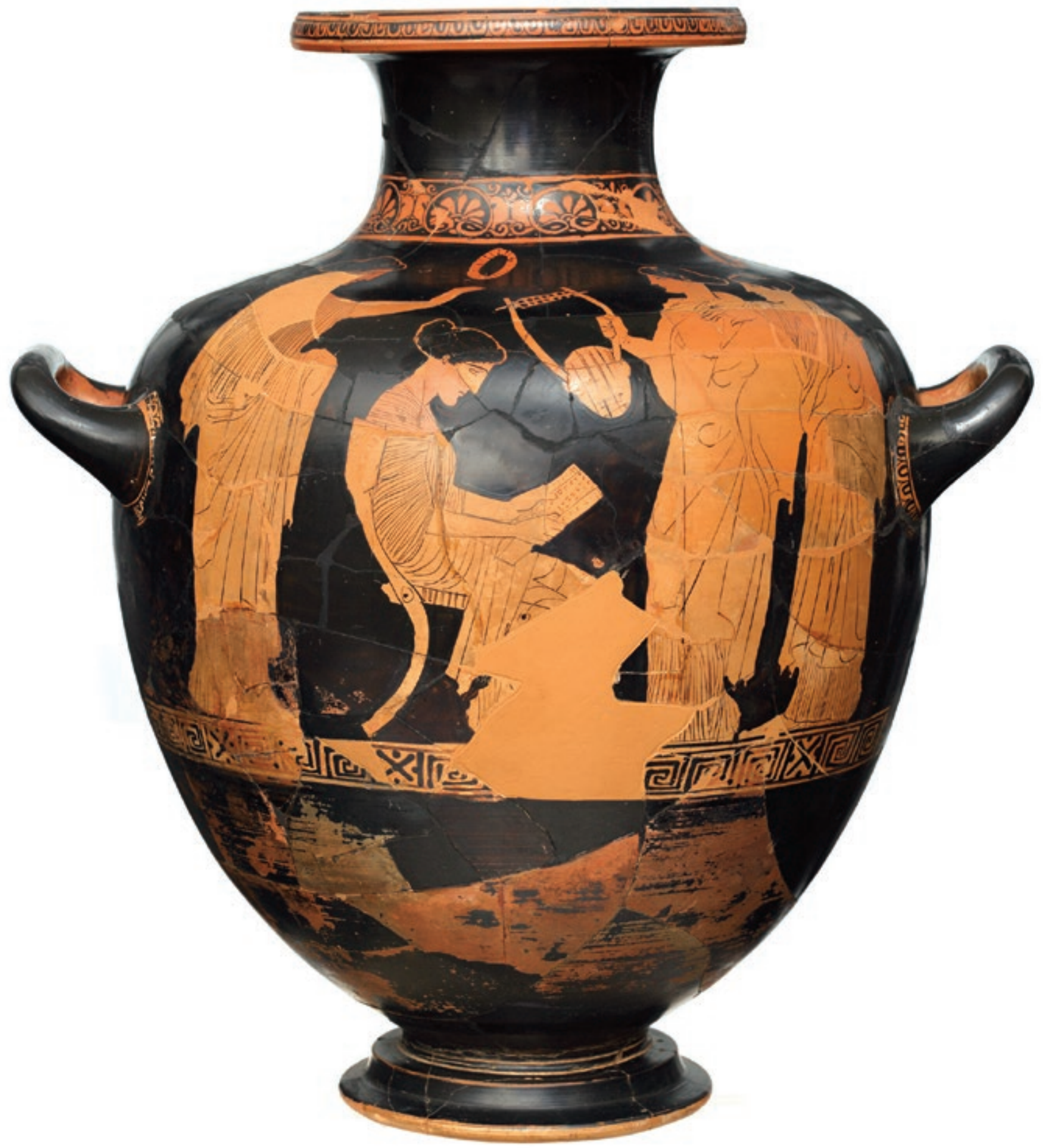

Hydrie à figures rouges, attribuée au Groupe de Polygnotos, 440-430 av. J.-C. (coll. Musée national archéologique d'Athènes, Inv. 1260) 


\section{LA FEMME AU ROULEAU : IMAGES DE FEMMES LETTRÉES DANS LE MONDE GREG ANTIQUE}

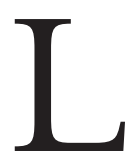

e livre antique était, avant tout, le livre en rouleau, appelé en grec byblos et en latin volumen. Présent en Égypte depuis le milieu du III ${ }^{\mathrm{e}}$ millénaire, le papyrus semble avoir été introduit en Grèce pendant le règne des pharaons saïtes philhellènes $\left(7^{\mathrm{e}} \mathrm{6}^{\mathrm{e}}\right.$ siècles avant J.-C.). Il est certain qu'au $5^{\mathrm{e}}$ siècle, l'usage des rouleaux de papyrus à Athènes est courant. Le papyrus servait aussi bien pour les comptes qu'à la mise par écrit des œuvres littéraires. Ce matériel, assez cher, était extrait d'une plante que l'on trouve en grande quantité dans les régions marécageuses au bord du Nil, principalement dans le delta. La seule description d'époque de la méthode de fabrication du papyrus nous est parvenue grâce à Pline l'Ancien, auteur du $1^{\text {er }}$ siècle après J.-C. (Histoire naturelle, 13.74-82). Le prix assez onéreux du papyrus le rendait peu accessible, aussi les gens utilisaient-ils, pour l'apprentissage des lettres, les comptes du quotidien et même la correspondance, des tessons de céramique appelés ostraka. Il arrivait toutefois, comme le montre la documentation retrouvée en Égypte, que le verso de papyrus, plus rêche que le recto où l'on écrivait dans le sens des fibres, fût utilisé pour les exercices d'écoliers. Ceux-ci allaient des simples alphabets que les petits écoliers essayaient de noter, en respectant l'ordre des lettres grecques, jusqu'aux formules plus élaborées comprenant des séquences de vers homériques. Les morceaux de papyrus servaient donc comme support d'apprentissage, alors qu'un rouleau standard, contenant environ 20 feuilles, était utilisé pour noter des écritures plus élaborées. C'est cette dernière fonction qui fait qu'à l'époque hellénistique, le rouleau commence à être associé, puis à signifier, à lui seul, l'éducation (paideia) de ceux dans les mains desquels il était représenté : hommes, mais aussi femmes.

\section{Une éducation grecque}

L'éducation, réservée en priorité aux garçons dont les parents avaient les moyens financiers pour payer un maître, n'était toutefois pas complètement inaccessible aux filles, surtout quand la cité se chargeait, en tant qu'organisme politique, de l'éducation de ses futurs citoyens. Ainsi, au $5^{\mathrm{e}}$ siècle avant J.-C., Hérodote raconte, dans son Enquête (5.27), le malheur qui s'était abattu sur les habitants de Chios quand le toit de l'école s'était écroulé sur les 120 enfants qui apprenaient leurs lettres et dont un seul réussit à s'échapper. Trois siècles plus tard, une inscription de Téos mentionne la fondation d'un riche citoyen, Polythrous, qui laissa un capital important dont les intérêts devaient servir à financer l'éducation des plus jeunes, filles et garçons (Sylloge 3, $\mathrm{n}^{\circ}$ 578). À Athènes même, réputée plus conservatrice que les autres cités, des peintures sur céramique attique représentent de jeunes écolières en train d'apprendre à lire et à écrire, avec des tablettes, des styli ou des rouleaux de papyrus, parfois déployés, sur lesquels on aperçoit des lettres.

Ces témoignages se heurtent à l'image stéréotypée et misogyne propagée par les auteurs classiques. Ainsi, selon Xénophon dans son traité d'économie domestique, l'éducation des filles devait entre mise, en principe, au service de son rôle de bonne ménagère : la femme d'Ischomaque, le protagoniste du traité de Xénophon (9.10), était capable à 14 ans de rédiger une liste des fournitures. Si, en accord avec ces principes, Théophraste conseille de donner aux filles une éducation lettrée pour qu'elles puissent mieux assumer leurs responsabilités domestiques, Ménandre affirme qu'éduquer une femme revient à « donner plus de venin à un serpent " (F 702). Finalement, l'alphabétisation des filles devait avoir lieu 
en fonction du milieu social, ces dernières étant parfois instruites en même temps que les garçons de la famille. Il est bien connu que les femmes qui avaient le plus facilement accès à l'éducation n'étaient pas les filles de bonne famille, mais bien les hétaïres, pour la plupart des étrangères, des métèques. La plus célèbre reste Aspasie, originaire de Milet, compagne de Périclès, dont on disait, dans le dialogue Ménéxenos de Platon, qu'elle avait formé entre autres à l'art oratoire son propre compagnon. Elle n'est pas la seule : on peut encore citer Nééra, héroïne d'une affaire judiciaire retentissante au 4 e siècle, ou Gnathaena, sa contemporaine, connue pour ses mots d'esprit.

En contraste avec l'Athènes classique, d'autres cités, notamment dans les îles, offrent une toute autre image. Le centre d'une éducation ouverte également aux femmes était, à l'époque archaïque, Mytilène, située sur l'île de Lesbos, d'où était originaire la poétesse Sappho $\left(6^{\mathrm{e}}\right.$ siècle avant J.-C.), représentante avec Alcée de la poésie lyrique. Issue d'une famille aristocratique, elle s'était mariée et avait eu une fille, Kléis. Sa poésie, des poèmes d'amour plaintifs, a été imitée notamment par le poète romain Catulle. Image même de l'intellectuelle grecque (voir ill. p. 16), elle ouvre la série des poétesses célèbres, notamment la Béotienne Corinne. Une élève de Sappho, Damophyla, fonda une école en Pamphylie, au sud-ouest de l'Asie Mineure.

\section{Les amies des Muses}

En accord avec l'image évoquée ci-dessus, on peut envisager deux directions de réflexion sur les rapports entre les femmes et les lettres en Grèce ancienne : elles sont souvent poétesses et toujours amies des Muses. Les patronnes de toute activité littéraire, artistique ou scientifique étant les Muses, il n'est pas rare que les femmes qui s'y exerçaient soient comparées à cellesci. Dans l'art grec, les Muses sont représentées en tant que lettrées, un rouleau ou une tablette à la main. Dans ce type iconographique, le rouleau n'est pas un attribut quelconque, mais bien le symbole de l'activité intellectuelle que la Muse est censée patronner ${ }^{1}$. C'est souvent ce même schéma qu'on retrouve pour les femmes lettrées, au point que l'on a parfois du mal à distinguer entre les déesses et leurs protégées : sur un rhyton de Nysa (Turkménistan), alors qu'on pensait voir des Muses, on a identifié parmi les dix femmes qui lisent, écrivent ou tiennent des instruments d'écriture, les poétesses Myrtis, Sappho et Corinne ${ }^{2}$. Une relation étroite est donc établie entre les Muses et leurs disciples, comme on le voit dans une célèbre peinture sur une pyxide conservée à Boston, où le poète Archiloque se présente aux Muses (voir ill. ci-contre). Sappho en particulier, mais aussi d'autres poétesses, étaient appelées " la dixième Muse ", le nombre canonique étant arrêté assez tôt à neuf.

Cette comparaison flatteuse, appliquée aux grands noms de la poésie, s'est par la suite étendue à la pratique des épigrammes funéraires que les proches érigeaient en l'honneur des jeunes filles ou de jeunes épouses disparues prématurément. Bien entendu, il faut dans ce dernier cas tenir compte de certaines règles qui régissent le style des épigrammes. Ce que nous percevons à travers les textes est la conception grecque de la femme idéale.

Les femmes se rangeaient en trois catégories, calquées sur des figures divines ou littéraires : Pénélope, pour valoriser leurs qualités d'épouse et de mère, puis Aphrodite et les Muses. Les femmes placées sous le signe des Muses ne sont pas très nombreuses, bien que leurs liens avec celles-ci s'expriment souvent dans les mêmes termes que pour les hommes : elles sont servantes des Muses ou amies des Muses. Qui plus est, dans le cas des femmes " amies des Muses ", il est plus plausible que les divinités soient présentes comme patronnes de la musique plutôt qu'en tant que protectrices des lettres : l'éducation musicale occupait davantage de place dans la formation des jeunes filles que dans celle des jeunes hommes, le mot paideia (éducation, culture) n'apparaissant pas dans les épitaphes féminines. La sagesse associée aux femmes n'est pas toujours une sophia littéraire, mais elle suggère davantage la modération et la bienséance (sôphrosynè). Cependant, pour qu'une femme soit réellement accomplie, il fallait qu'à côté des vertus féminines traditionnelles, elle incarne des valeurs complémentaires que l'on trouve dans l'« œuvre des Muses ". Dans l'épigramme funéraire de Procla de Cyrène, d'époque impériale, on voit s'étaler tous les éléments contribuant à l'accomplissement d'une femme : "Bien connue pour ma beauté, mon intelligence et ma gaieté, ma bonne naissance et ma sagesse, les ouvrages magnifiques des femmes, métiers et corbeilles, les leçons des Muses savantes, la discrétion de ma vie, la retenue de mes pensées (...) ${ }^{3}$. La même image se dégage d'une épigramme en provenance de Panticapée, capitale du Royaume du Bosphore au nord du Pont-Euxin. Le père de la jeune Théophilè (originaire de Sinope), accablé par la douleur, la plaint en ces mots : " Tu avais reçu une beauté enviée par les mortels, toi la dixième Muse, une Grâce mûre pour le mariage, modèle de pudeur " ${ }^{4}$. 


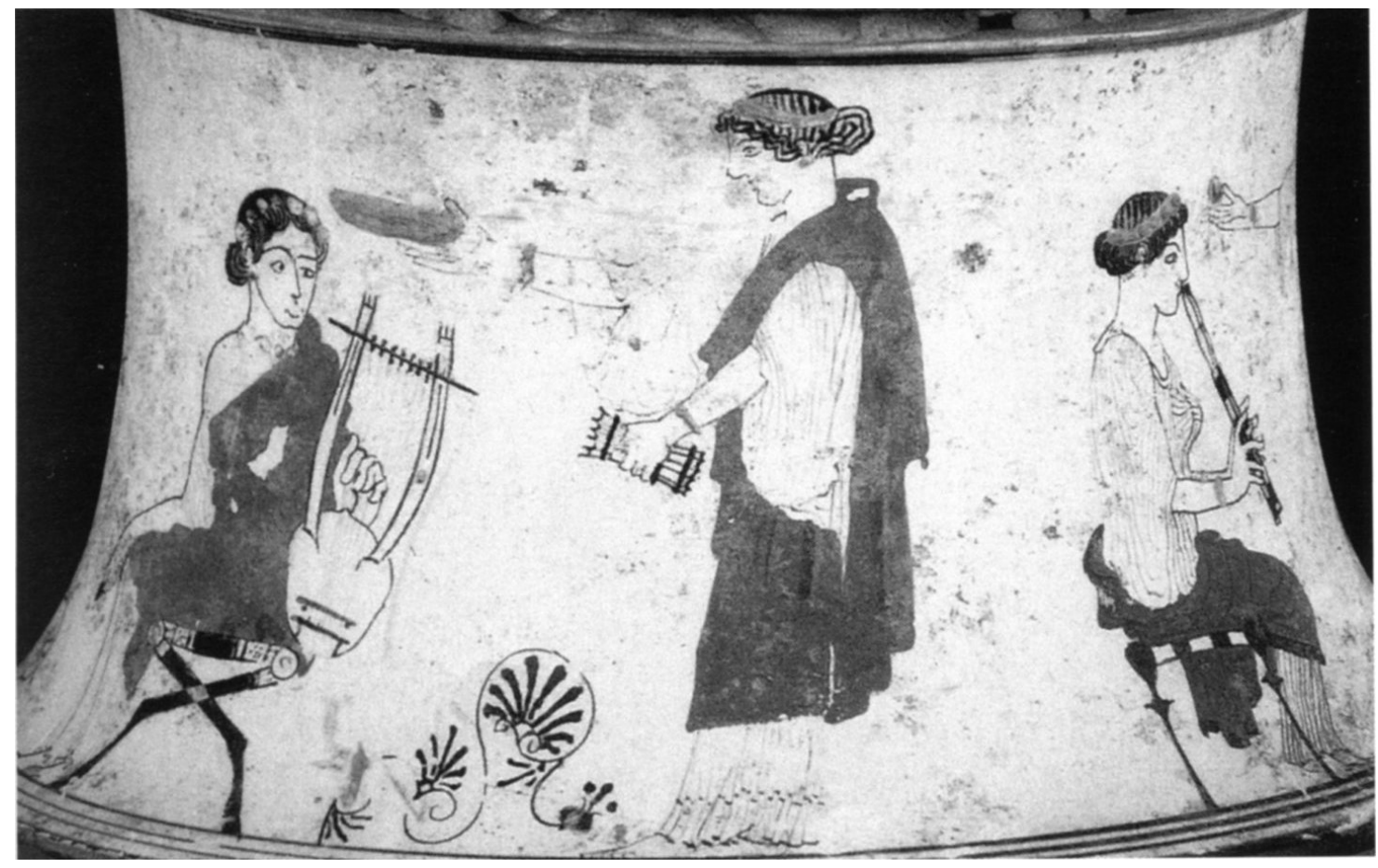

Pyxide à figures blanches, 46o-450 av. J.-C., Boston, Museum of Fine Arts. D’après M. Papini, « La dolce rugiada delle Muse ", dans A. Bottini (éd.), Musa pensosa. L'immagine dell'intellettuale nell'antichità, Milan, 2006, p. 49, fig. 9 


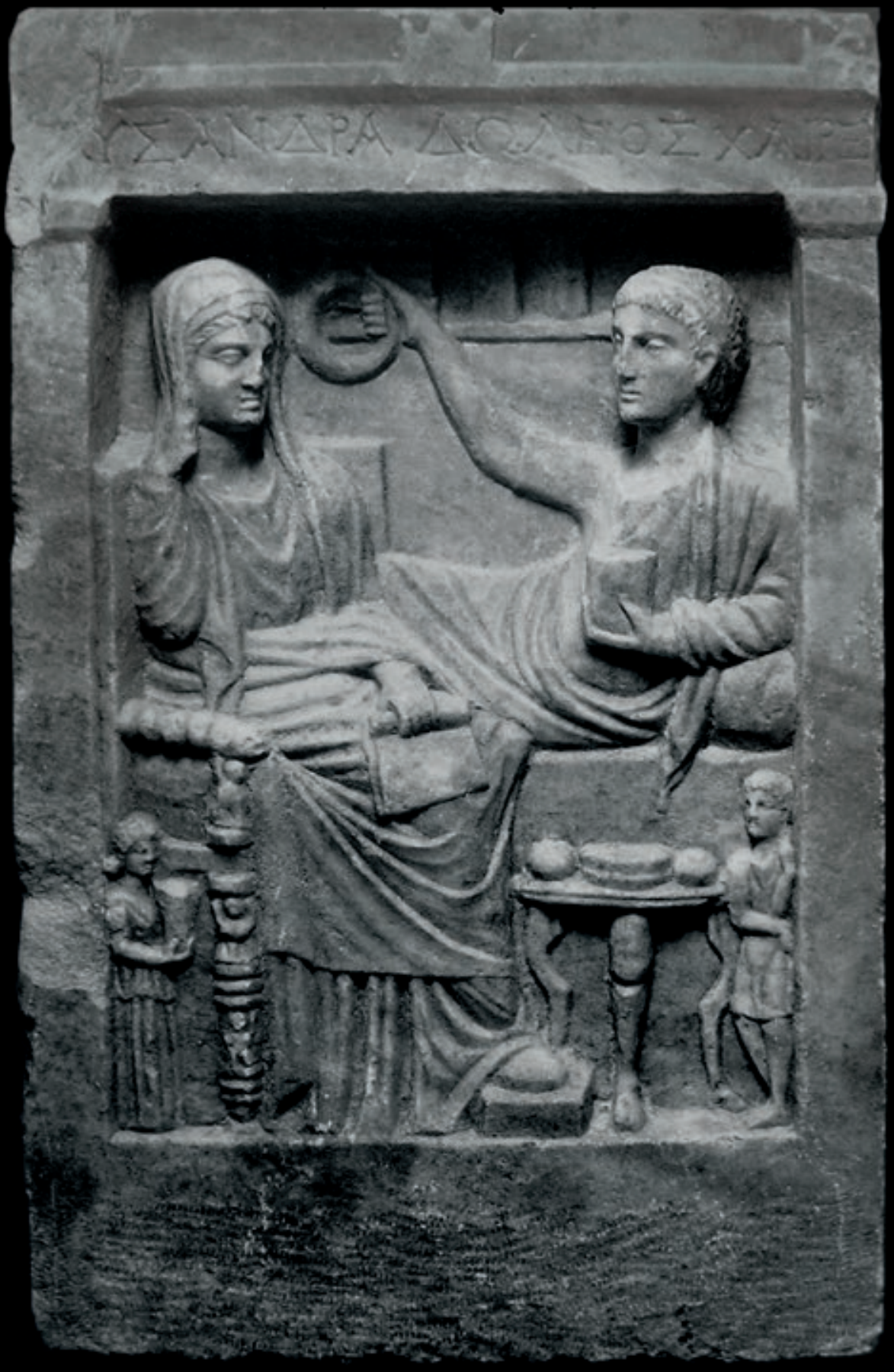

Stèle funéraire de marbre. D’après G. Koch, Roman Funerary Sculpture. Catalogue of the Collections. The Paul Getty Museum, Malibu (California), 1988, n 33, p. 93 


\section{Le rouleau de papyrus dans la main des femmes}

Dans une grande majorité des cas, l'équivalent de la référence aux Muses dans le texte de l'épigramme est le rouleau de papyrus qui figure dans la main des défunts ou dans le champ de la stèle funéraire. L'association entre les Muses et le rouleau de papyrus, comme symbole de l'éducation, est corroborée par un certain nombre de stèles où l'image et le texte s'appuient réciproquement.

On a depuis longtemps identifié ce que l'on peut appeler, sans toutefois en faire un cliché, la représentation spécifique du lettré, le volumen à la main ${ }^{5}$. P. Zanker met en avant la notion de l'" intellectualisation " du portrait du citoyen dans l'imagerie de la basse époque hellénistique. Comme le montre plus récemment L. Del Corso, le volumen trouve une place stable dans le contexte du nouveau paradigme iconographique élaboré au cours $\mathrm{du} 3^{\mathrm{e}}$ siècle avant J.-C. pour représenter les " professionnels " des lettres. C'est à l'époque hellénistique qu'est réalisée une longue série de portraits d'intellectuels, faciles à distinguer en raison de certains éléments récurrents : un mode particulier de s'habiller, certains gestes, l'expression du visage, la posture du corps. À ces éléments s'ajoute la présence des livres, point commun de l'apparence des philosophes, grammairiens, poètes et orateurs. En même temps que s'opère une pénétration de l'image canonique de la lecture - personnage en train de lire un rouleau à moitié déroulé, ou bien avec une tablette à la main - dans les arts dit " mineurs " et sur les scènes de banquets funèbres, au jeune homme en train de lire s'ajoute au $2^{\mathrm{e}}$ siècle avant J.-C. le motif de la jeune fille s'adonnant à cette même activité 6 . On peut même dire que si, à l'époque hellénistique, le rouleau est synonyme d'une culture à laquelle pouvaient aspirer aussi les gens modestes, il est également le symbole d'une révolution de genre. Ainsi, l'éducation apparaît comme une qualité universelle pour commémorer les défunts, indépendamment de leur origine sociale ou de leur sexe.

Certes, les femmes apparaissent dans une majorité de cas associées à leurs maris dans les activités de lecture, sur des stèles funéraires à banquets. L'homme, allongé sur un lit de banquet (klinè), tient d'une main un rouleau de papyrus à moitié déroulé, dans l'attitude de la lecture interrompue. La femme, assise sur le bout de la klinè ou sur une chaise, soutient d'une main son voile dans une attitude de pudeur, tandis que dans l'autre main elle tient à son tour un rouleau ou prend une tablette que lui tend un esclave, situation illustrée par une stèle funéraire de Byzance de la fin de l'époque hellénistique ${ }^{7}$. Cependant, on voit dans une scène de couple, toujours de Byzance, que ce n'est pas l'homme, ni les deux époux, mais la femme seule qui est représentée le rouleau à la main. Qui plus est, seul le nom de la femme est inscrit : Lysandra, fille de Dôlès ( $1^{\text {er }}$ siècle après J.-C.). Assise sur un siège d'honneur comme ceux du théâtre, la défunte tient de la main gauche un volumen de grandes dimensions, partiellement déroulé sur ses genoux (voir ill. ci-contre).

J. Fabricius insiste sur la position particulière des épouses de Byzance, lettrées comme leurs maris, mais s'interroge aussi sur la valorisation qui en découle pour la famille ${ }^{8}$. Pour comprendre la présence sur les stèles de femmes seules, accompagnées de rouleaux, il convient de placer la stèle dans son contexte. Une célèbre stèle érigée à la fin du $2^{\mathrm{e}}$ siècle avant J.-C. à Sardes, en l'honneur de Ménophila (voir ill. p. 23), constitue un exemple d'autant plus précieux qu'il reste singulier dans le monde grec. À gauche, on voit des rouleaux retenus par des liens et une corbeille à laine ; en dessous de ces objets est gravée la lettre alpha (le chiffre 1) ; une fleur de lys est représentée en haut à droite. L'épigramme offre un véritable code de déchiffrement de l'image : "Une élégante et précieuse femme, voici ce que montre cette pierre. Qui est-elle ? Les écrits des Muses le révèlent : Ménophila [...] Le livre indique sa sagesse, ce qu'elle porte sur la tête évoque sa magistrature, le chiffre 1 révèle qu'elle était enfant unique, le panier à laine est signe de sa vertu bien ordonnée, le lys témoigne de sa jeunesse ". Le texte affirme que la défunte possédait la sophia, accordée par les Muses, et établit une relation entre cette sophia féminine et les rouleaux de papyrus ${ }^{9}$. Cette sagesse n'est pas la sôphrosynè qui consistait à savoir gérer sa maison et élever sa progéniture, mais bien celle que les livres confèrent. Ménophila était fille unique : sa famille avait mis tous ses espoirs dans cet enfant, qui cumule les droits - y compris celui à la paideia - et les obligations d'un héritier mâle.

\section{Professionnelles du savoir ou femmes de métier}

Il existe toutefois des situations où le rouleau, en plus d'être un indice de l'éducation ou de l'intérêt pour les lettres, représente le véritable symbole d'un métier. C'est le cas de la femme-médecin (iatreinè) Mousa de Byzance, qui fait exception dans l'iconographie de sa cité. Elle est la seule à ne pas être représentée dans la scène typique de couple, mais debout, dans la posture du lettré, le rouleau à la main (voir ill. p. 24). La relation entre son nom, Muse, 
son métier et le rouleau qu'elle tient ne saurait être fortuite. C'est en effet un " nom de plume " (Berufsname), et on peut être certain, quand on pense que la transmission du savoir médical se faisait dans le cadre familial, qu'elle venait d'une famille de praticiens. Le nom s'accorde avec la présence du rouleau qui renvoie à la détention par cette femme de l'art (technè) médical. Ses compétences étaient sans doute plus étendues que celles d'une simple sage-femme (maia), même si l'exercice de sa profession s'inscrivait dans une sphère d'activité très proche. Les femmes-médecins étaient amenées à donner aux femmes leurs soins pour les faire accoucher et aussi à prodiguer ceux-ci aux nourrissons. Une " gynécologue " antique était un médecin généraliste dévolu principalement à une clientèle féminine et aux enfants. On le voit sur une stèle funéraire d'Athènes, érigée pour la " sage-femme et médecin " Phanostratè, au milieu du $4^{\mathrm{e}}$ siècle avant J.-C. ${ }^{10}$ Elle est représentée assise, la main tendue en direction d'une jeune femme debout à droite et entourée de quatre enfants d'âges différents.

Les femmes pouvaient jouer également un rôle dans la théorisation de la médecine, car plusieurs sont citées comme auteurs de traités, dans le domaine de la pharmacopée. Une femme considérée comme une égale des grands médecins de son époque est Antiochis, qui vécut au $1^{\text {er }}$ siècle avant J.-C. Galien cite un médecin célèbre, Asclépiadès de Bithynie, qui la considèrait comme une autorité pour l'œdème, la sciatique, l'arthrite. Il affirme aussi qu'une autre célébrité, le médecin Héracleidès de Tarente, aurait écrit un livre sur les hémorragies du nez dédié à Antiochis ${ }^{11}$. Les auteurs de compilations de l'Antiquité tardive attribuent plusieurs traités de gynécologie à des femmes : Cléopatra à l'époque hellénistique et Métrodôra au $5^{e}$ siècle après J.-C. Les femmes possédaient donc une certaine forme de savoir, concernant notamment le corps féminin et les soins corporels, qui se retrouvait dans des traités écrits par elles.

Le rouleau accompagne également les poétesses. Selon Pausanias (Description de la terre, 2.20.8), dans le temple d'Aphrodite situé dans l'agora d'Argos, on pouvait voir de son temps une stèle figurant la poétesse Télésilla, aux pieds de laquelle étaient représentés des rouleaux de papyrus. Ceux-ci étaient sans aucun doute un symbole de son métier et non seulement de ses intérêts intellectuels : ils étaient censés contenir son œuvre. La tradition n'a malheureusement conservé que peu de fragments appartenant aux poétesses célèbres, au point que certaines, qui jouissaient d'une grande réputation dans l'Antiquité, sont presque ignorées de la plupart de la critique moderne. C'est le cas de Moirô de Byzance, épouse d'un philologue et mère d'un poète tragique portant le nom expressif d'Homéros. Ce dernier, qui faisait pourtant partie de la Pléiade, fut victime comme sa mère de l'anonymat causé par la perte de ses œuvres. Il est mentionné, précisément en rapport avec sa mère, par le poète égyptien tardif Christodôros de Coptos, qui fait la différence entre cet Homéros et son célèbre homonyme : " c'était plutôt celui que, près des rivages de la Thrace, enfanta sa mère, la célèbre Moirô de Byzance, à qui les Muses enseignèrent dès son enfance les lois de la poésie héroïque ". Moirô est citée par le poète Antipatros de Thessalonique (Anthologie palatine, IX, 26) parmi les neuf poétesses canoniques, dont le nombre répond à celui des neuf Muses: "Ces femmes aux divins accents, l'Hélicon les a nourries de chants, ainsi que le rocher macédonien de Piérie : Praxilla, Moirô, l'éloquente Anytè, l'Homère des femmes, Sappho, parure des Lesbiennes aux belles boucles, Érinna, l'illustre Télésilla et toi, Corinne, qui chantas l'impétueux bouclier d'Athéna, Nossis aux accents de femme et Myrtis douce à entendre, qui toutes produisirent des pages éternelles ". Le portrait de Moirô aurait été réalisé par Céphisodote, fils du sculpteur Praxitèle, vers 300 avant J.-C.

Parmi les métiers de l'intellect exercés par les femmes, on peut également citer celui d'historien. Un personnage tout à fait remarquable est Aristodama de Smyrne (fin du $3^{\mathrm{e}}$ siècle avant J.-C.). Elle avait composé des poèmes qui concernaient l'histoire des Étoliens, sous la forme d'une chronique contemporaine et d'un récit mythique sur les origines de ce peuple. La cité de Lamia, en Thessalie, et celle de Chaleion, en Locride, lui avaient octroyé notamment le droit de cité, privilège exceptionnel pour une femme ${ }^{12}$, acquis seulement grâce à ses compétences. Une autre historienne, Nicoboulè, est l'auteur d'un ouvrage historique sur la vie quotidienne et sur la mort d'Alexandre le Grand. À l'instar de nombreux historiens dont les œuvres sont perdues à jamais, Nicoboulè fut victime du véritable naufrage de la littérature hellénistique contemporaine du Conquérant ou de peu postérieure. Elle est la seule femme parmi les auteurs qui ont relaté la geste d'Alexandre ${ }^{13}$. Enfin, d'autres femmes ont gravité dans les cercles philosophiques - par exemple, l'Académie de Platon - ou ont été attirées par les sciences de l'érudition, comme Agallis de Corcyre, élève d'Aristophane de Byzance, et Hestiaia d'Alexandrie de Troade, qui s'occupèrent de la critique homérique. 


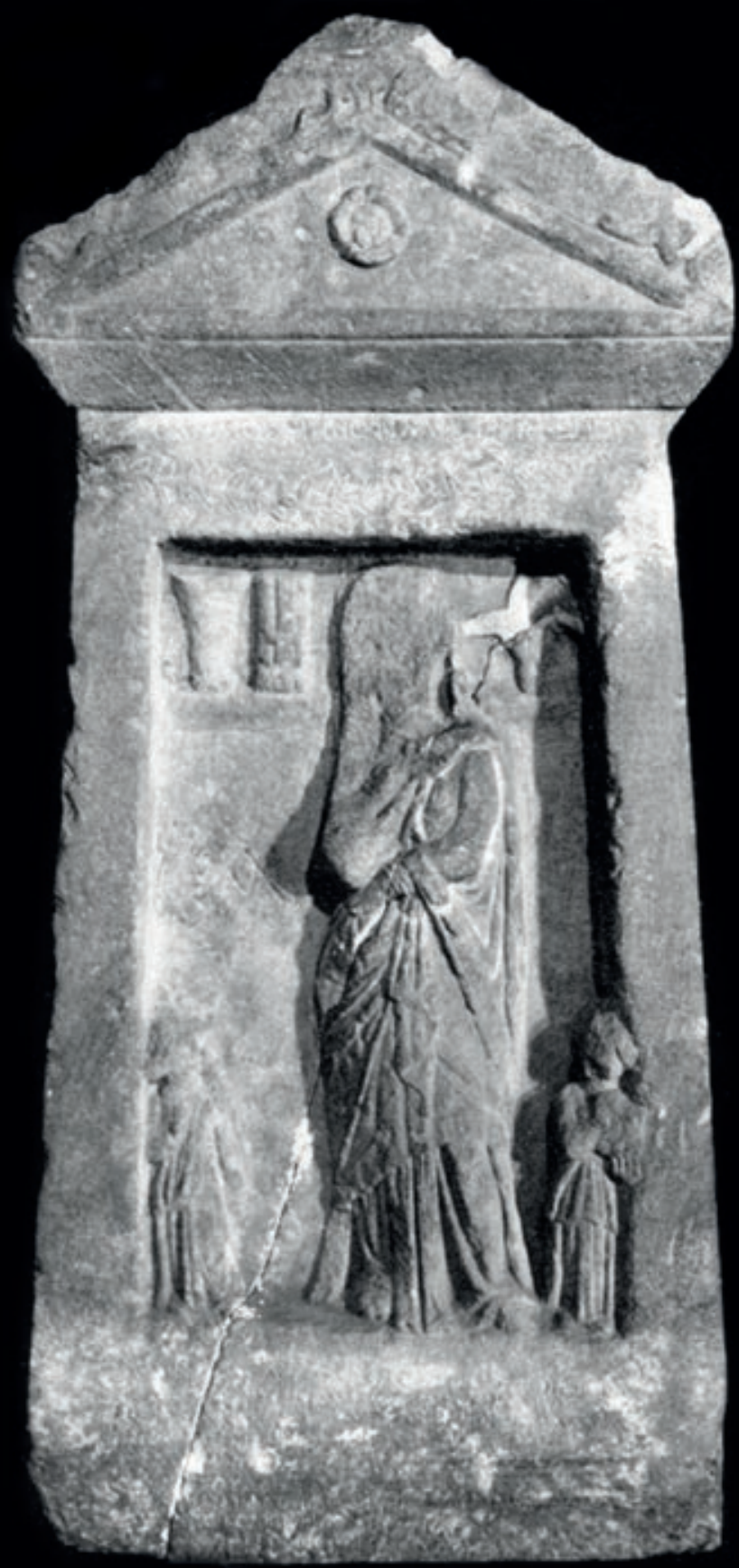

Stèle funéraire de marbre. D’après E. Pfuhl, H. Möbius, Die ostgriechische Grabreliefs, I, Mayence, 1977, $\mathrm{n}^{\circ} 418, \mathrm{pl} .69$ 


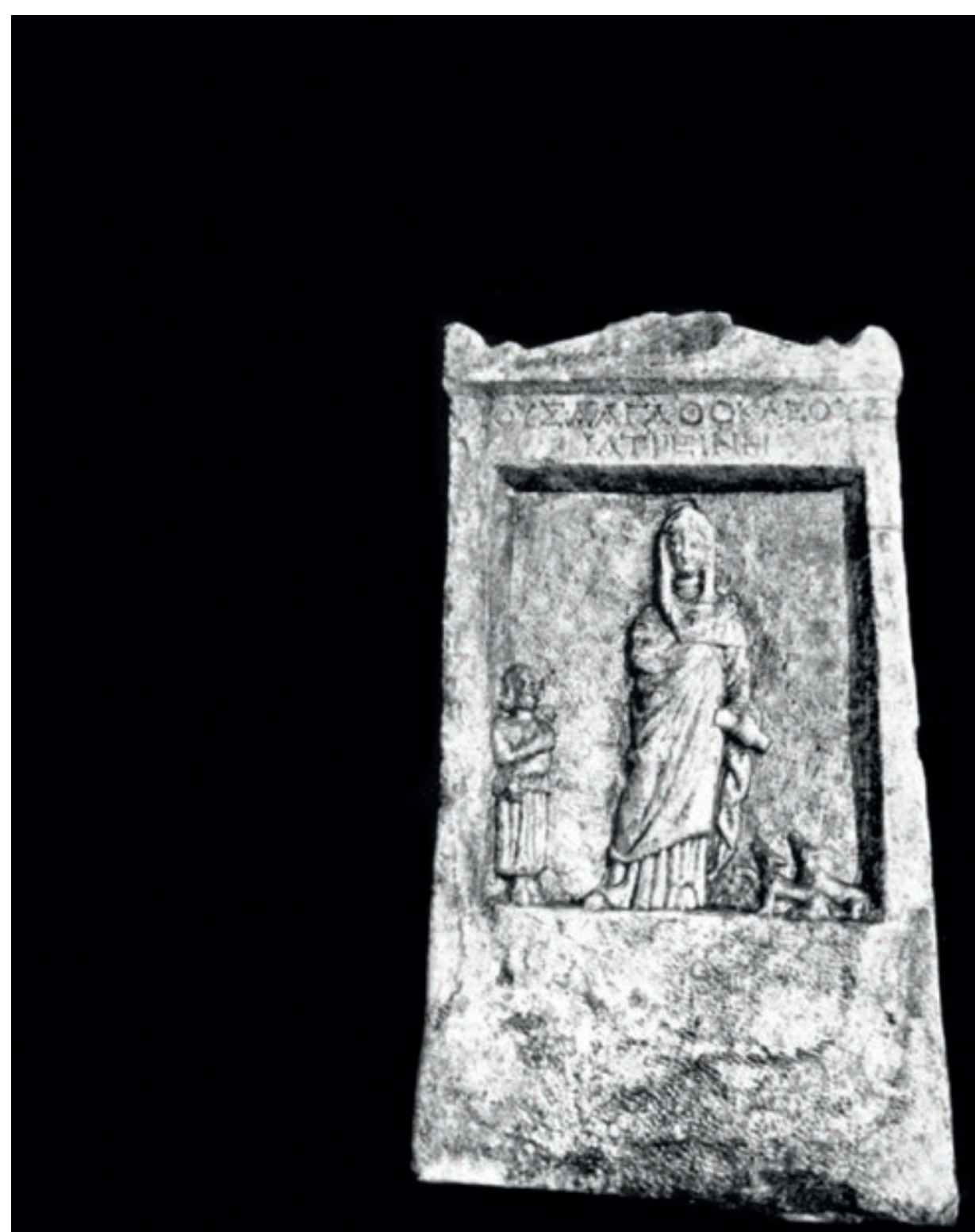

Stèle funéraire de marbre. D’après E. Pfuhl, H. Möbius, Die ostgriechische Grabreliefs, I, Mayence, 1977, n 467, pl. 77 
Femmes éduquées, femmes lettrées, femmes intellectuelles : les portraits que nous avons présentés vont de la femme instruite dans le cadre familial jusqu'à la professionnelle du savoir, auteur de traités médicaux, de poèmes ou de chroniques historiques. Dans un monde grec qui change et s'étend avec la conquête d'Alexandre jusqu'aux frontières de l'Inde, portant ainsi la paideia au rang de valeur hellénique par excellence, l'histoire des femmes lettrées évolue elle aussi. Elle s'écrit non pas en subsidiaire de celle de leurs confrères masculins, mais bien en parallèle de celle-ci, et entremêlée à elle.

\section{Madalina Dana}

\section{Notes}

1 - L. Del Corso, " Libro e lettura nell'arte ellenistica. Note storico-culturali ", in Segno e testo, 4, 2006, p. 74-76

2 - P. Bernard, «Les rhytons de Nysa. I. Poétesses grecques », in JS, 1985 , p. $43-49$

3 - C. Dobias-Lalou, "Une épigramme funéraire de Cyrène ", in REG, 95, 1982, p. 37-53 (avec traduction)

4 - W. Peek, Griechische Vers-Inschriften, Berlin, 1955, $\mathrm{n}^{\circ} 1989$. Pour d'autres exemples, voir M. Dana, Culture et mobilité dans le Pont-Euxin. Approche régionale de la vie culturelle des cités grecques, Bordeaux, 2011, p. 150-152.

5 - P. Zanker, The Mask of Socrates. The Image of the Intellectual in Antiquity, Berkeley-Los Angeles-Oxford, 1995

6 - L. Del Corso, art. cit., p. 85-106

7 - E. Pfuhl, H. Möbius, Die ostgriechische Grabreliefs, II, Mayence, 1979, $\mathrm{n}^{\circ} 2035$, pl. 295

8 - J. Fabricius, Die hellenistischen Totenmahlreliefs. Grabrepräsentation und Wertvorstellungen in ostgriechischen Städten, Munich, 1999, p. 274-275

9 - A. Bielman, "Une vertu en rouleau ou comment la sagesse vint aux Grecques ", in R. Frei-Stolba, A. Bielman, O. Bianchi (éd.), Les femmes antiques entre sphère privée et sphère publique. Actes du Diplôme d'études avancées. Universités de Lausanne et Neuchâtel, 2000-2002, Berne, 2003, p. 79-81, avec traduction.

10- E. Samama, Les médecins dans le monde grec. Sources épigraphiques sur la naissance d'un corps médical, Genève, 2003, p. 109-110, $\mathrm{n}^{\circ} 2$

11 - Galien, XIII.250 et 341 K ; Galien, XII.691 K. Voir M. Dana, "Femmes et savoir médical dans les mondes antiques ", in A. Gargam (éd.), Femmes de sciences de l'Antiquité au XIX' siècle. Réalités et représentations, Dijon, 2014, p. 24-27.

12- M. Dana, " La poétesse épique Aristodama de Smyrne ", in V. Sébillotte-Cuchet, S. Boehringer (éd.), Hommes et femmes dans l'Antiquité gréco-romaine, Paris, 2011, p. 98-101

13 - S. Cagnazzi, Nicobule e Panfila : frammenti di storiche greche, Bari, 1997, p. 9-28 